\title{
Abordagem terapêutica para a prevenção das complicações da doença hepática gordurosa não alcoólica em obesos: revisão narrativa
}

\author{
Therapeutic approach for the prevention of complications of non-alcoholic fatty liver \\ disease in obese people: a narrative review
}

\section{Enfoque terapéutico para la prevención de complicaciones de la enfermedad del hígado graso no alcohólico en personas obesas: una revisión narrativa}

Mateus Camargos Silva Alves Simão ${ }^{1 *}$, Caio Aguiar Caires², Clara Jamarino Braga de Almeida1, Igor Martins Costa ${ }^{1}$, Igor Nascimento Alves ${ }^{3}$, Isabela de Oliveira Resende Neves ${ }^{1}$, Izabella Moreira Fulgêncio $^{1}$, Laryssa Mara Vieira Moreira ${ }^{4}$, Mariana de Fátima Lage Amaral ${ }^{5}$, Victor Campos Guimarães'.

\section{RESUMO}

Objetivo: Discutir, através de uma revisão narrativa, a prevenção das complicações da Doença Hepática Gordurosa Não Alcoólica (DHGNA) em pacientes obesos e apresentar as possíveis intervenções terapêuticas. Revisão bibliográfica: A DHGNA representa uma manifestação da obesidade e dos componentes da síndrome metabólica, associada ao sedentarismo e aos hábitos alimentares inapropriados, que são caracterizados pela alta ingestão calórica. $O$ tratamento consiste primordialmente na mudança de estilo de vida, tendo como pilares fundamentais hábitos alimentares e a atividade física. As principais opções terapêuticas descritas na literatura são mudança de estilo de vida, terapia farmacológica e cirurgia bariátrica. A terapia medicamentosa é indicada para todos os pacientes por reduzir a progressão da doença. Os medicamentos que mostraram melhora significativa na fibrose com eficácia elevada e segurança a longo prazo são pioglitazona e liraglutida, enquanto a maioria dos outros fármacos permanecem com baixos níveis de evidência. Considerações Finais: O manejo terapêutico mais eficaz da DHGNA baseia-se na alimentação adequada e na prática regular de atividade física a longo prazo.

Palavras-chave: Doença hepática gordurosa não alcoólica, Esteatose hepática, Exercício físico; Obesidade; Terapêutica.

\begin{abstract}
Objective: discuss, through a narrative review, the prevention of Non-alcoholic Fatty Liver Disease (NAFLD) complications in obese patients and present possibilities for therapeutic interventions. Literature Review: NAFLD represents a manifestation of obesity and the components of the metabolic syndrome, associated with a sedentary lifestyle and inappropriate eating habits, characterized by high caloric intake. The treatment consists mainly in lifestyle interventions based on dietary habits and exercises. The main therapeutic options described in literature are lifestyle changes, pharmacological therapy and bariatric surgery. Drug therapy is indicated for all patients because it reduces the progression of the disease. The drugs which had shown significant improve in fibrosis with high efficiency and long-term safety are pioglitazone e liraglutide, meanwhile
\end{abstract}

\footnotetext{
${ }_{1}^{1}$ Pontifícia Universidade Católica de Minas Gerais (PUC-MG), Betim - MG.

*E-mail: camargosmateus@gmail.com

2 Pontifícia Universidade Católica de Minas Gerais (PUC-MG), Betim - MG.

${ }^{3}$ Centro Universitário Barão de Mauá (CBM), Ribeirão Preto - SP.

${ }^{4}$ Centro Universitário Governador Ozanam Coelho (UNIFAGOC), Ubá - MG.

${ }^{5}$ Universidade Iguaçu (UNIG), Itaperuna - RJ.
} 
most of other drugs maintain a lower level of evidence. Final considerations: the most efficient therapeutic management to NAFLD is based on proper diet and regular practice of exercises in a long-term basis.

Keywords: Non-alcoholic fatty liver disease; Fatty liver; Exercise; Obesity; Therapeutics.

\section{RESUMEN}

Objetivo: Discutir, a través de una revisión narrativa, la prevención de complicaciones de Enfermedad del Hígado Graso no Alcohólico (EHGNA) en pacientes obesos y presentar posibles intervenciones terapéuticas. Revisión Bibliográfica: La EHGNA representa una manifestación de la obesidad y de los componentes del síndrome metabólico, asociado con un estilo de vida sedentario y hábitos alimenticios inapropiados, que se caracterizan por una alta ingesta calórica.El tratamiento consiste principalmente en un cambio en el estilo de vida, basado en hábitos alimenticios y actividad física. Las principales opciones terapéuticas descritas en la literatura son los cambios en el estilo de vida, la terapia farmacológica y la cirugía bariátrica. La terapia con medicamentos está indicada para todos los pacientes porque reduce la progresión de la enfermedad.Los medicamentos que mostraron una mejora significativa en la fibrosis con alta eficiencia y seguridad a largo plazo son la pioglitazona y la liraglutida, mientras que la mayoría de los otros medicamentos permanecen con bajos niveles de evidencia. Consideraciones finales: El manejo terapéutico más efectivo de EHGNA se basea en una nutrición adecuada y actividad física regular a largo plazo.

Palabras-clave: Enfermedad del hígado graso no alcohólico; Hígado graso; Ejercicio físico; Obesidad; Terapéutica.

\section{INTRODUÇÃO}

A Doença Hepática Gordurosa Não Alcoólica (DHGNA) pode ser definida como a evidência de mais de $5 \%$ de esteatose hepática por exame de imagem ou histológico associado à inexistência de causas secundárias para acúmulo de gordura no fígado, como consumo de álcool, uso prolongado de medicação esteatogênica ou causa genética. (CHALASANI N, et al., 2018). A DHGNA é uma doença multifatorial e estima-se que a prevalência de indivíduos obesos com esta hepatopatia corresponde a 51,34\% (YOUNOSSI ZM, et al., 2016).

A obesidade é definida como a ocorrência de Índice de Massa Corporal (IMC) maior ou igual a $30 \mathrm{~kg} / \mathrm{m}^{2} \mathrm{e}$ constitui um fator de risco marcante também para Esteato Hepatite Não-Alcoólica (NASH) (ABESO, 2016). A DHGNA representa uma manifestação da obesidade e dos componentes da Síndrome Metabólica (SM), associada ao sedentarismo e aos hábitos alimentares inapropriados, que são caracterizados pela alta ingestão calórica (LISBOA CQ, et al., 2016). A obesidade, dislipidemia e diabetes Mellitus tipo II são os fatores de risco mais frequentes. Esses se associam à SM que é caracterizada pela presença de três ou mais das seguintes condições: obesidade central (aumento da gordura no abdome), hipertensão arterial, dislipidemia e diabetes (SOLER, 2008).

O início da DHGNA é caracterizado pelo acúmulo de triglicerídeos intracelular, devido ao desequilíbrio existente entre a sua síntese e sua a degradação. A obesidade, nesse contexto, surge como um fator contribuinte para o desequilíbrio, estimulando a lipólise tecidual e gerando um progressivo aumento de lipídeos nos hepatócitos. Além disso, o crescimento dos estoques de triglicerídeos podem atingir níveis tóxicos provocando o estresse oxidativo e consequentemente a produção de radicais livres associado a lesão mitocondrial (SOLER, et al., 2008).

Uma clínica norte americana constatou uma incidência anual de hepatocarcinoma (HCC) de 0,52\% em pacientes com NASH, e de 0,04\% em pacientes com DHGNA (ASCHA et al., 2010). Um estudo belga constatou que a SM estava presente em 14\% dos pacientes com DHGNA e em 38\% dos pacientes com $\mathrm{NASH}$. Além disso, 23,9\% dos pacientes com DHGNA e diabetes evoluíram com cirrose hepática e 19\% morreram por doença hepática, para os não diabéticos com DHGNA esses números foram $10,6 \%$ e $2 \%$, respectivamente (FRANCQUE et al., 2011). 
Como tem origem multifatorial e fatores reversíveis, a terapêutica inclui desde o tratamento não farmacológico à terapia medicamentosa e cirúrgica (POLYZOS AS, et al., 2017). A terapia não farmacológica, que corresponde a mudança do estilo de vida (MEV), é a primeira linha de tratamento da DHGNA, sendo recomendada para todos os pacientes que apresentam o quadro, por ser capaz de reduzir a progressão da doença (JEZNACH-STEINHAGEN A, et al., 2019).

Concomitantemente ao MEV, é importante que os componentes da síndrome metabólica associada ao quadro sejam tratados. Como a adesão e manutenção do MEV podem ser difíceis para alguns pacientes, a introdução de medicamentos torna-se necessária. A principal finalidade da terapia farmacológica é reduzir a inflamação hepática e a esteatose, diminuindo os níveis de aminotransferases, melhorando o perfil lipídico do paciente e contribuindo para redução da balonização dos hepatócitos (SANYAL AJ, et al., 2010).

O objetivo do presente artigo é discutir, por meio de uma revisão narrativa, a prevenção das complicações da DHGNA em pacientes obesos e expor as possíveis intervenções terapêuticas que possam ocasionar uma melhora clínica desses pacientes.

\section{REVISÃO BIBLIOGRÁFICA}

O tratamento da Doença Hepática Gordurosa Não Alcoólica tem como finalidade a redução da resistência à insulina e do estresse oxidativo, por meio do controle das condições sabidamente associadas como a obesidade, a dislipidemia e o Diabetes Mellitus (DM). Além disso, o tratamento também objetiva reduzir a inflamação e fibrose do fígado (LISBOA CQ, et al., 2016). As principais opções terapêuticas descritas na literatura são MEV, terapia farmacológica e cirurgia bariátrica (LISBOA CQ, et al., 2016).

\section{Mudança no estilo de vida}

A terapia não farmacológica, baseada na MEV, ou seja, perda de peso a partir da adequação da dieta e atividade física constitui a principal estratégia para o manejo da DHGNA. Evidências epidemiológicas sugerem a relação direta de esteatose hepática não alcoólica e um estilo de vida não saudável (ELHENCE $A$, SHALIMAR, 2020).

A dieta mediterrânea é uma opção para pacientes com DHGNA, pois é rica em fibras e ácidos graxos poliinsaturados, além disso, outra alternativa é a dieta low-carb. Ambas podem gerar certos benefícios para o quadro, auxiliando na redução da resistência à insulina e na melhora da esteatose. A dieta low-carb, por exemplo, é capaz de reduzir o perfil lipídico, tendo em vista a sua baixa relação com doenças cardiovasculares e, também, a maior facilidade em adesão que alguns estudos vêm mostrando. Essas opções de alimentação quando associadas a atividades físicas diárias e ao processo de aconselhamento e acompanhamento individual, em um curto intervalo de tempo, pode possibilitar ajustes que irão favorecer a manutenção dos efeitos benéficos a longo prazo (ELHENCE A, SHALIMAR, 2020).

Além da redução calórica com perda de peso, é preconizada a restrição da ingestão de gordura, pois aumenta os níveis de triglicerídeos, colesterol e estresse oxidativo, que atuam no processo inflamatório dos hepatócitos. Uma dieta rica em carboidratos e pobre em proteínas é um dos fatores que predispõem a DHGNA. Dessa maneira, a ingestão de carboidratos simples também deve ser restrita e os açúcares excluídos da dieta, pois podem promover alterações metabólicas, como lipogênese e depósito de triglicerídeos nos hepatócitos (JEZNACH-STEINHAGEN A, et al., 2019).

Há indícios que o consumo de café também ajuda na redução da esteatose e prevenção da fibrose e que os pacientes com DHGNA que apresentam um consumo moderado de álcool podem apresentar progressão da fibrose hepática, sendo assim, estes devem ser desestimulados a ingerir quantidades alcoólicas abusivas (EKSTEDT M, et al., 2009).

Em relação à prática de exercícios físicos, um estudo randomizado caso-controle contou com 31 participantes obesos ou com sobrepeso, com DHGNA comprovada por biópsia. Os participantes desse estudo foram alocados em dois grupos: um que recebeu MEV intensa, com dieta e exercício físico, e outro que recebeu apenas educação em saúde, que era o controle. Após 48 semanas, ao reavaliar esses pacientes, os que receberam MEV intensa perderam $9,3 \%$ do peso e diminuíram a esteatose hepática em pelo menos 3 
pontos do score de lesão hepática, enquanto o controle emagreceu $0,2 \%$ do peso corporal e diminuíram a esteatose em 2 ou menos pontos. Os participantes que conseguiram alcançar a meta de perda de peso do estudo (de 7\%) comparados com os que perderam menos do que a meta, tiveram melhora significativa da esteatose e inflamação lobular (PROMRAT, et al., 2009).

Outro estudo randomizado de caso-controle acompanhou por seis meses 203 participantes com DHGNA moderada a grave, confirmada por exame de ultrassonografia. Eles foram divididos em dois grupos, o primeiro seguiu um programa de atividade aeróbica moderada de 30 minutos por dia, 5 vezes por semana. $O$ segundo grupo realizou treino aeróbico com adição de musculação, com duração de 60 minutos, pelo menos 3 vezes por semana. O score de DHGNA utilizado pelo estudo reduziu em $22 \%$ nos pacientes que realizaram apenas atividade aeróbica, ao passo que o grupo que associou a musculação os resultados foram menos efetivos, embora também tenha sido benéfico no tratamento de DHGNA. (FRANCO, et al., 2019).

Há uma concordância entre os autores de que a MEV consiste na base do tratamento da DHGNA, tendo seus benefícios comprovados. Portanto, a adequação da dieta e a atividade física devem ser estimuladas em todos os pacientes com essa hepatopatia.

\section{Tratamento farmacológico}

O tratamento farmacológico é indicado para pacientes que não conseguiram uma redução significativa de peso ou que apresentaram uma evolução da esteatose na biópsia (JEZNACH-STEINHAGEN A, et al., 2019). Deve ser considerado em pacientes com Esteato Hepatite Não Alcoólica com fibrose moderada e estágios mais avançados, ou pacientes com rápida piora da fibrose e com alto risco de progressão da doença. Não é recomendado o tratamento farmacológico para fibrose hepática em pacientes com DHGNA ou quadro inicial de NASH (SUMIDA Y e YONEDA M, 2018).

As drogas antidiabéticas se mostraram eficientes no tratamento da DHGNA (JEZNACH-STEINHAGEN A, et al., 2019). As pioglitazonas pertencem à classe das tiazolidenedionas e atuam por meio da ligação aos receptores nucleares peroxisome proliferator-activated receptors (PPARs) do tipo y presentes no tecido adiposo, endotélio, macrófago, nas células beta e hematopoiéticas. Através deles, induzem à transcrição de genes relacionados ao metabolismo glicídico e lipídico que aprimoram a sensibilidade do indivíduo à insulina aumenta a captação de glicose periférica e reduz a gliconeogênese no fígado (JEZNACH-STEINHAGEN A, et al., 2019; GOMES MB, 2006).

Essas drogas têm benefícios descritos em diversos artigos quando empregada em pacientes com DHGNA. Um estudo randomizado indicou que a pioglitazona reduz as aminotransferases, a esteatose, a balonização dos hepatócitos e a inflamação, porém não contribui com os desfechos primários da esteatose e, em pacientes diabéticos, apresenta melhora da fibrose (SANYAL AJ, et al., 2010). Os efeitos adversos descritos foram ganhos de peso e retenção hídrica. $O$ tratamento a longo prazo com pioglitazona é seguro e efetivo em pacientes com pré-diabetes, Diabetes Mellitus tipo 2 ou NASH (CUSI K, et al., 2016).

A rosiglitazona também reduz a esteatose e as aminotransferases, melhora a resistência insulínica, eleva os níveis de adiponectina, porém produz cãibras e edema de membros inferiores (RATZIU V, 2008). A saroglitazona reduz os níveis de triglicerídeos, aminotransferases e gordura hepática, aperfeiçoa a resistência insulínica sem os efeitos adversos da pioglitazona, mas pode causar astenia e gastrite (ELHENCE A e SHALIMAR, 2020).

A respeito das glitazonas, os autores concordam com seus benefícios em relação ao perfil lipídico dos pacientes, principalmente a pioglitazona, e alertam acerca dos efeitos colaterais, que devem ser levados em consideração na escolha terapêutica.

A liraglutida, um incretinomimético, aumenta a secreção pós-prandial de insulina, reduz a secreção de glucagon pós prandial e promove a saciedade em pacientes com Diabetes Mellitus tipo 2 (JEZNACHSTEINHAGEN A, et al., 2019). Em um ensaio clínico, foi possível observar que a liraglutida diminui os níveis de aminotransferases, o peso e a esteatose, em relação ao tratamento da NASH, evita a progressão da fibrose (ARMSTRONG MJ, et al., 2016). Os benefícios desta medicação em pacientes diabéticos com DHGNA é unânime entre os autores. 
Os inibidores da dipeptidil peptidase 4 (DPP-4), assim como os análogos de Peptídeo Semelhante ao Glucagon 1 (GLP-1) representados pela liraglutida, atuam potencializando o efeito da incretina. Os inibidores da DPP-4 bloqueiam a ação dessa enzima, aumentando os níveis de GLP-1, que potencializam a secreção de insulina, diminuem os níveis de glicose no sangue, reduzem a produção de glucagon e retardam o esvaziamento gástrico. Pacientes com DHGNA tendem a apresentar uma alta atividade da DPP-4, o que pode justificar a administração de inibidores dessa enzima no tratamento da doença (OHKI T, et al., 2012).

Em pacientes diabéticos tipo 2 com DHGNA, a sitagliptina promoveu o controle da doença, diminuição de alanina aminotransferase (ALT), gama GT e colesterol. Porém, peso, IMC, aspartato aminotransferase (AST) e fibrose não se alteraram. Já a liraglutida promoveu redução de peso, IMC, enzimas hepáticas, inflamação e fibrose, além do melhor controle diabético. Com pioglitazona, houve bom controle diabético, redução de triglicérides, enzimas hepáticas, fibrose e melhora de inflamação, mas houve aumento no ganho de peso e no IMC (OHKI T, et al., 2012).

O estudo de Ohki T, et al. (2012) concluiu que a redução das enzimas hepáticas ocorreu nos três grupos, mostrando que o tratamento de Diabetes Mellitus tipo 2 com esses hipoglicemiantes interfere de maneira positiva na inflamação dos hepatócitos em pacientes com DHGNA. Visto que a obesidade é um dos principais fatores de risco para o desenvolvimento da DHGNA e que o tratamento medicamentoso é indicado para pacientes que não obtiveram perda de peso significativa com a MEV, a liraglutida mostrou-se superior à sitagliptina no tratamento de DHGNA em pacientes com DM tipo 2, pois promove maior redução do peso e redução da fibrose hepática.

A metformina é um outro antidiabético capaz de reduzir a gliconeogênese hepática, aumentar a captação de glicose periférica além de causar redução das aminotransferases, do peso e da esteatose. Entretanto, o uso desta medicação não tem sido observado em pacientes com DHGNA devido a ausência de benefícios histológicos significativos (LI Y, et al., 2013).

Os citoprotetores, como ácido obeticólico e a pentoxifilina, podem vir a ser úteis no tratamento da DHGNA. O ácido obeticólico é um ácido lipofílico sintético agonista do receptor de ácido biliar que reduz a gliconeogênese, lipogênese e melhora a sensibilidade insulínica (ELHENCE A e SHALIMAR, 2020). Este ácido pode regular o metabolismo de lipídios, reduzir a inflamação e a fibrose no fígado (JEZNACHSTEINHAGEN A, et al., 2019). O ácido obeticólico melhorou de forma significativa a fibrose e os principais componentes da atividade da doença NASH e mostram melhora histológica clinicamente, com probabilidade razoável de prever benefício clínico (YOUNOSSI ZM, et al., 2019).

Acerca da pentoxifilina, trata-se de um inibidor de fosfodiesterase e do TNF- $\alpha$ que reduz a inflamação dos hepatócitos (ELHENCE A e SHALIMAR, 2020). A pentoxifilina reduz níveis de fibrose no fígado, ou seja, é capaz de promover uma melhora dos parâmetros histológicos. Entretanto, mais estudos precisam ser realizados para determinar a sua eficácia ao longo do tempo (ZEIN CO, et al., 2011).

Outros medicamentos que têm a capacidade de readequar o perfil lipídico são aramchol, estatinas e orlistat e, por isso, pondera-se que eles possam vir a ser estratégias terapêuticas. O aramchol é um inibidor da enzima estearoil-CoA dessaturase e reduz a síntese de ácidos graxos monoinsaturados e elevando a beta oxidação destes (ELHENCE A e SHALIMAR, 2020). A terapia com aramchol pode ser usada no tratamento da esteatose, pois é uma droga segura, bem tolerada e reduz significamente a gordura hepática de pacientes com DHGNA. A redução da gordura acumulada no fígado com o uso de aramchol foi dose dependente e demonstrou melhora da disfunção metabólica presente na DHGNA (SAFADI R, et al., 2014).

Em um ensaio clínico, pacientes com suspeita de DHGNA que fizeram o uso estatina apresentaram melhora substancial nos testes hepáticos e redução dos eventos cardiovasculares. A estatina mostrou-se segura na abordagem terapêutica da DHGNA (ATHYROS VG, et al., 2010).

$O$ orlistat é um remédio anti-obesidade que melhora os parâmetros bioquímicos ao inibir a enzima lipase gastrointestinal (ELHENCE A e SHALIMAR, 2020). Em ensaio clínico, o orlistat reduziu os níveis de aminotransferases, gama GT, glicose, triglicerídeos, além de diminuir a resistência insulínica e o IMC, mas não demonstrou melhora da fibrose do fígado. Reitera-se que o orlistat não é o medicamento de primeira escolha no tratamento de DHGNA e NASH (WANG H, et al., 2018). 
Em outro ensaio clínico, o uso de frutooligossacarídeos por pacientes com avanço da DHGNA para NASH, na dose de $8 \mathrm{~g}$ duas vezes ao dia, demonstrou considerada redução dos níveis séricos de AST, discreta melhora de ALT e resposta estatisticamente insignificante de triglicerídeos. Ao exame de ultrassom, não houve mudanças na morfologia e constituição do fígado. Dessa forma, o uso do medicamento, associado à outras terapias mais eficazes, pode ter efeito positivo no tratamento da função hepática em pacientes com DHGNA, não sendo indicado seu uso como monoterapia (DAUBIOUL CA, et al., 2005).

O selonsertib é um anti apoptótico que inibe a enzima quinase 1, responsável por regular a apoptose, é uma das opções para o manejo da fibrose e da apoptose hepática. Em pacientes com NASH com moderada fibrose hepática, o selonsertib demonstrou redução da fibrose, da gordura hepática e diminuição do risco de cirrose. O uso do medicamento em pacientes com Esteato Hepatite Não Alcoólica estado mais avançado com fibrose grave ainda está sendo testados (JEZNACH-STEINHAGEN A, et al., 2019). Os pacientes que receberam selonsertib demonstraram melhora na diminuição da fibrose, diminuição da progressão para cirrose, redução da rigidez hepática e dos percentuais de gordura do fígado (SUMIDA Y e YONEDA M, 2018).

O ácido ursodesoxicólico (UDCA) é um agente anti-inflamatório citoprotetor que pode ser utilizado no tratamento de doenças do fígado (NAKAJIMA, 2012). O uso de UDCA mostrou redução nos índices de ALT, diminuição dos níveis de fibrose e melhora da resistência insulínica ao longo prazo em pacientes com NASH (RATZIU, 2010). Os ácidos graxos poliinsaturados n-3 de cadeia longa (PUFAs) reduzem triglicerídeos plasmáticos, pressão arterial, inflamação e melhora a função vascular (NAKAJIMA K, 2012). Além do efeito de prevenção da DHGNA, a suplementação dietética com PUFAs de cadeia longa $n-3$ reduz os níveis de gordura hepática nos portadores da doença (SHAPIRO H, et al., 2010).

Os antioxidantes, em especial a vitamina E, podem vir a ser grandes aliados no tratamento, uma vez que o estresse oxidativo é um mecanismo de progressão da doença causando lesões hepatocelulares de maior gravidade (LAVINE JE, et al., 2011). A vitamina E, em um ensaio clínico randomizado, demonstrou redução das aminotransferases e melhora dos parâmetros histológicos como balonização, esteatose e inflamação, mas não interfere na fibrose (SANYAL AJ, et al., 2010). Há preocupações no uso a longo prazo pelo risco elevado de acidente vascular cerebral hemorrágico e câncer de próstata (LASSAILLY G, et al., 2016).

Um ensaio clínico randomizado triplo cego, encontrou resultados positivos para o uso de probióticos no tratamento de DHGNA em crianças e adolescentes obesos. O tratamento com administração de um mix de probióticos contendo Lactobacillus acidophilus, Bifidobacterium lactis, Bifidobacterium bifidum e Lactobacillus rhamnosus associado a com MEV foi adicionado ao grupo controle. Após 12 semanas, ultrassonografia e exames laboratoriais mostraram melhora significativa dos níveis de ALT, AST e de circunferência abdominal. Além disso 17 pacientes apresentaram níveis de gordura no fígado normais (antes classificados estágios I e II de DHGNA) após uso dos probióticos, contra 5 do grupo placebo (FAMOURI F, et al., 2017).

Produtos naturais foram identificados com efeitos benéficos em modular o metabolismo de gorduras e em melhorar doenças hepáticas gordurosas, dentre eles, flavonoides, alcaloides e saponinas (ZHU ZJ, et al., 2020). Esses agentes fitoterápicos podem não necessariamente serem utilizados como suplementos, mas incluídos na dieta, participando da MEV. O uso de probióticos, vitamina $\mathrm{E}$ e fitoterápicos chamam a atenção como terapias alternativas coadjuvantes ao tratamento clínico, em pacientes com essa preferência, estas terapias auxiliares devem ser consideradas.

\section{Cirurgia bariátrica}

A cirurgia bariátrica, ou gastroplastia, é recomendada para obesos com Índice de Massa Corporal (IMC) acima de $40 \mathrm{~kg} / \mathrm{m}^{2}$ ou acima de $35 \mathrm{~kg} / \mathrm{m}^{2}$ associado a comorbidades (ELHENCE A e SHALIMAR, 2020). Embora não seja uma opção terapêutica para a DHGNA, esta abordagem atua diretamente na obesidade que é uma das principais causas de base dessa hepatopatia. Estima-se que cerca de $95 \%$ dos obesos submetidos à cirurgia bariátrica têm DHGNA (SOUVEEK; ARKA; ABHIJIT, 2020). Comprova-se através de um estudo prospectivo não randomizado que existe uma perda de peso entre 14 a $25 \%$ do peso em 10 anos, em qualquer que seja a forma de cirurgia escolhida. Essa redução de peso, objetivo da cirurgia bariátrica, além de contribuir para remissão do DM e para queda nos eventos cardiovasculares, contribui, também, para evitar as possíveis complicações da DHGNA (SJÖSTRÖM L, et al., 2004). 
Uma meta-análise incluiu 15 estudos e 766 biópsias hepáticas pareadas de pacientes submetidos à cirurgia bariátrica e mostrou melhora significativa de diversos componentes da DHGNA: redução da esteatose em 93\%, redução da esteato-hepatite em 82\% e redução de fibrose em 73\% (MUMMADI RR, et al., 2008).

Portanto, a escolha dessa terapêutica não deve levar em consideração uma patologia exclusiva e sim todo o contexto do paciente. A abordagem multidisciplinar nesse cenário é necessária para elencar os malefícios e benefícios desta terapêutica para o paciente.

\section{CONSIDERAÇÕES FINAIS}

A modificação do estilo de vida é a peça chave do tratamento da Doença Hepática Gordurosa Não Alcoólica. A implementação de uma dieta adequada e a prática de exercícios físicos regulares favorecem a perda de peso que refletem na melhora da histologia hepática. O manejo farmacológico com a pioglitazona e com a liraglutida mostrou eficácia elevada e segurança a longo prazo e, por isso, podem ser usadas quando a meta de perda de peso não foi alcançada pelo paciente ou dependendo do estágio da Esteato Hepatite Não Alcoólica. Embora existam várias drogas em estudo para o tratamento de DHGNA, a maioria possui benefício incerto. A cirurgia bariátrica é uma opção a ser avaliada individualmente, dosando os benefícios e malefícios dessa terapia para o indivíduo, não sendo indicada para tratamento exclusivo dessa comorbidade. Por fim, são necessários mais estudos para avaliação dos benefícios das terapias farmacológicas associadas à MEV no tratamento da DHGNA.

\section{REFERÊNCIAS}

1. ARMSTRONG MJ, et al. Liraglutide safety and efficacy in patients with non-alcoholic steatohepatitis (LEAN): A multicentre, double-blind, randomised, placebo-controlled phase 2 study. Lancet. 2016; 387: 679-690.

2. ASCHA, Mustafa S. et al. The incidence and risk factors of hepatocellular carcinoma in patients with nonalcoholic steatohepatitis. Hepatology. 2010; 51(6): 1972-1978.

3. ASSOCIAÇÃO BRASILEIRA PARA O ESTUDO DA OBESIDADE E DA SÍNDROME METABÓLICA (ABESO). Associação Brasileira para o Estudo da Obesidade e da Síndrome Metabólica. Diretrizes Brasileiras de Obesidade, 4.ed. São Paulo: ABESO, 2016.

4. ATHYROS VG, et al. Safety and efficacy of long-term statin treatment for cardiovascular events in patients with coronary heart disease and abnormal liver tests in the Greek Atorvastatin and Coronary Heart Disease Evaluation (GREACE) Study: a post-hoc analysis. The Lancet Conference Alerts. 2010; 376 (1): 1916-1922.

5. CHALASANI N, et al. The diagnosis and management of nonalcoholic fatty liver disease: Practice guidance from the American Association for the Study of Liver Diseases. Hepatology. 2018; 67 : 328-357.

6. CUSI K. Long-term pioglitazone treatment for patients with nonalcoholic steatohepatitis and prediabetes or type 2 diabetes mellitus: a randomized trial. Annals of Internal Medicine. 2016;165: 305-15.

7. DAUBIOUL CA, et al. Effects of oligofructose on glucose and lipid metabolism in patients with nonalcoholic steatohepatitis: results of a pilot study. European Journal of Clinical Nutrition. 2005; 59(5): 723-726.

8. EKSTEDT M, et al. O consumo de álcool está associado à progressão da fibrose hepática na doença hepática gordurosa não alcoólica. Scandinavian Journal of Gastroenterology. 2009; 44: 366-374.

9. ELHENCE A, SHALIMAR. Treatment of non-alcoholic fatty liver disease - Current perspectives. Indian Journal of Gastroenterology: Official Journal of the Indian Society of Gastroenterology. 2020.

10. ESLAM M, et al. Genetics and epigenetics of NAFLD and NASH: Clinical impact. Journal of Hepato-Biliary-Pancreatic Sciences. 2018; 68(2): 268-279.

11. FAMOURI F, et al. Effects of Probiotics on Nonalcoholic Fatty Liver Disease in Obese Children and Adolescents. Journal of Pediatric Gastroenterology and Nutrition. 2017; 64(3): 413-417.

12. FRANCO I, et al. Effectiveness of two physical activity programs on non-alcoholic fatty liver disease. a randomized controlled clinical trial. Revista de La Facultad de Ciencias Médicas de Córdoba, 76(1), 26.27 fev 2019.

13. FRANCQUE, S. et al. High prevalence of advanced fibrosis in association with the metabolic syndrome in a Belgian prospective cohort of NAFLD patients with elevated ALT. Results of the Belgian NAFLD registry. Acta Gastroenterologica Belgica. 2011; 74(1): 9-16.

14. FUKUHARA T, et al. Efficacy and safety of sitagliptin for the treatment of nonalcoholic fatty liver disease with type 2 diabetes mellitus. Hepatogastroenterology. 2014; 61(130): 323-328.

15. GOMES MB. Glitazonas e síndrome metabólica: mecanismos de ação, fisiopatologia e indicações terapêuticas. Arq Bras Endocrinol Metab. 2006; 50(2): 271-280.

16. JEZNACH-STEINHAGEN A, et al. Dietary and Pharmacological Treatment of Nonalcoholic Fatty Liver Disease. Medicina (Kaunas). 2019; 55(5): 166.

17. LASSAILLY G, et al. Perspectives on Treatment for Nonalcoholic Steatohepatitis. Gastroenterology. 2016; 150(8), 1835-1848. 
18. LAVINE JE, et al. Effect of Vitamin E or Metformin for Treatment of Nonalcoholic Fatty Liver Disease in Children and Adolescents. JAMA. 2011; 305(16), 1659.

19. LI Y, et al. Metformin in non-alcoholic fatty liver disease: A systematic review and meta-analysis. Biomed Rep. 2013; 1(1): 57-64.

20. 20.. LISBOA CQ, et al. Current management of non-alcoholic fatty liver disease. Revista Associação Médica Brasileira. 2016; 9(62): 872-878.

21. MUMMADI RR, et al. Effect of bariatric surgery on nonalcoholic fatty liver disease:: systematic review and metaanalysis.. : systematic review and meta-analysis. Clinical Gastroenterology and Hepatology. 2008; 6(12): $1396-1402$.

22. NAKAJIMA K. Multidisciplinary Pharmacotherapeutic Options for Nonalcoholic Fatty Liver Disease. Int $\mathrm{J}$ Hepatol. 2012; 32: 80-91.

23. OHKI T, et al. The Effectiveness of Liraglutide in Nonalcoholic Fatty Liver Disease Patients with Type 2 Diabetes Mellitus Compared to Sitagliptin and Pioglitazone. ScientificWorldJournal. 2012.

24. POLYZOS AS, et al. Adipose tissue, obesity and non-alcoholic fatty liver disease. Minerva Endocrinologica. 2017; 42(2): 92-108.

25. PROMRAT, K; et al. Randomized controlled trial testing the effects of weight loss on nonalcoholic steatohepatitis. Steatohepatitis/Metabolic Liver Disease. American Association of the Study of Liver Diseases. 23 dec 2009.

26. RATZIU V, et al. A randomized controlled trial of high-dose ursodesoxycholic acid for nonalcoholic steatohepatitis. Journal Of Hepatology. 2011; 54(5): 1011-1019.

27. RATZIU V, et al. Rosiglitazone for Nonalcoholic Steatohepatitis: one-year results of the randomized placebo-controlled fatty liver improvement with rosiglitazone therapy (flirt) trial. Gastroenterology. 2008; 135(1): 100-110.

28. SAFADI R, et al. The fatty acid-bile acid conjugate Aramchol reduces liver fat content in patients with nonalcoholic fatty liver disease. Clinical Gastroenterology and Hepatology. 2014;12(12): 2085-2091.

29. SANYAL AJ, et al. Pioglitazone, vitamin E, or placebo for nonalcoholic steatohepatitis. The New England Journal of Medicine. 2010; 362: 1675-1685.

30. SHAPIRO $\mathrm{H}$, et al. The therapeutic potential of long-chain omega-3 fatty acids in nonalcoholic fatty liver disease. Clinical Nutrition. 2011; 30(1): 6-19.

31. SJÖSTRÖM L, et al. Lifestyle, diabetes, and cardiovascular risk factors 10 years after bariatric surgery. The New England Journal of Medicine. 2004; 351(26): 2683-2693.

32. SOLER GLN, et al. Doença Hepática Gordurosa Não-Alcoólica: associação com síndrome metabólica e fatores de risco cardiovascular. Rev. SOCERJ. 2008; 21(2): 94-100.

33. SUMIDA Y, YONEDA M. Current and future pharmacological therapies for NAFLD/NASH. Journal of Gastroenterology. 2018; 53(3): 362-376.

34. TAVARES FL, et al. Doença Hepática Gordurosa Não Alcoólica - Diagnóstico e tratamento: uma revisão de literatura. Pará Research Medical Journal. 2019; 3(2): 1-6.

35. WANG H, et al. Efficacy of orlistat in non-alcoholic fatty liver disease: A systematic review and meta-analysis. Biomed Rep. 2018; 9(1): 90-96.

36. YOUNOSSI ZM, et al. Global epidemiology of nonalcoholic fatty liver disease-Meta-analytic assessment of prevalence, incidence, and outcomes. Hepatology. 2016; 64: 73-84.

37. YOUNOSSI ZM, et al. Obeticholic acid for the treatment of non-alcoholic steatohepatitis: interim analysis from a multicentre, randomised, placebo-controlled phase 3 trial. Lancet. 2019; 394(10215): 2184-2196.

38. ZEIN CO, et al. Pentoxifylline improves nonalcoholic steatohepatitis: a randomized placebo-controlled trial. Hepatology. 2011; 54(5): 1610-1619.

39. ZHU ZJ, et al. Fatty liver diseases, mechanisms, and potential therapeutic plant medicines. Chinese Journal of Natural Medicines. 2020; 18(3): 161-168.

40. ZINMAN B, et al. Empagliflozin, Cardiovascular Outcomes, and Mortality in Type 2 Diabetes. The New England Journal of Medicine. 2015; 373(22): 2117-2128. 